\title{
Drug-induced encephalopathy in cervical cancers treated with ifosfamide
}

\author{
I Nyoman Gede Budiana \\ Melinda Febiani \\ Pande Kadek Aditya Prayudi \\ Division of Gynecologic Oncology, Obstet- \\ rics and Gynecology Department, Faculty of \\ Medicine, Udayana University Sanglah General \\ Hospital \\ Bali, Indonesia
}

\begin{abstract}
Ifosfamide, whether in single or combination regimen, have been used in many centers for end-stage cervical cancer cases or recurrent cases. Although it has shown satisfying results, especially in cases of platinum resistance, this drug is related to various side effects, such as encephalopathy. In this study, several cases of acute encephalopathy in cervical cancer patients treated with ifosfamide chemotherapy are described. This study is a retrospective study using all cervical cancer cases receiving ifosfamide chemotherapy in Sanglah Hospital Denpasar from 2015 to 2017. The diagnosis of encephalopathy was established by neurologists. Data obtained from hospital medical records. There were five patients who received the second line ifosfamide-cisplatin chemotherapy regimen at Sanglah Hospital Denpasar from 2015 to 2017. They had received first-line chemotherapy in form of 6 series of paclitaxel carboplatin and some of them received additional therapy in the form of external radiation and brachytherapy. Ifosfamide-cisplatin chemotherapy was given from one to six series, depending on the patient's response. Side effects in the form of encephalopathy appeared in four patients, while one patient managed to recover. Ifosfamide encephalopathy is a side effect that needs to be monitored. It has varied symptoms, but the symptoms are generally mild. Analysis of patient risk factors, patient education, and preparation for the management of encephalopathy should be performed in all cases that will receive ifosfamide. Methylene blue and thiamine can be used as a prophylactic and therapeutic choice for this condition.
\end{abstract}

Keywords: ifosfamide, cervical cancer, encephalopathy, methylene blue, thiamine

\section{Introduction}

Cervical cancer ranks the second most common cause of death due to cancer in women which causes 275,000 deaths each year. In America, in 2016, cervical cancer ranks the third most common gynecological cancer, with 12,990 new cases, and caused 4,120 deaths. The incidence of cervical cancer in Southeast Asia reaches 16.6 per 100,000 women, and Indonesia ranks fourth with 17 per 100,000. ${ }^{3}$ Research in 2012 in Indonesia shows that cervical cancer is the most common gynecological cancer, with 20,928 new cases per year. ${ }^{1}$

In early-stage cervical cancer, surgery with or without adjuvant chemotherapy or radiotherapy is the main choice of treatment. In advanced stages or in recurrent cases, radiotherapy and/ or chemotherapy is preferred. Single regimen cisplatin-based chemotherapy is used in most centers. However, the recurrent cases are resistant to platinum-based agents, causing unsatisfactory responses in some cases. Ifosfamide, both as a single regimen and a combination has now been used in many centers and shows a satisfactory response. ${ }^{2}$

However, the use of ifosfamide is related to several side effects such as bone marrow suppres- 
sion, bladder, and kidney toxicity, and nervous system dysfunction.

The neurotoxicity caused by ifosfamide can range from cranial nerve paralysis to acute encephalopathy and posterior reversible encephalopathy syndrome (PRES). ${ }^{3}$ In this study, we describe a series of cases of encephalopathy found in patients with cervical cancer who received ifosfamide chemotherapy.

\section{Case Report}

This is a retrospective study conducted at Sanglah Hospital Denpasar, Bali, Indonesia. This hospital is a national referral center for Eastern Indonesia, which serves the management of various gynecologic oncology cases. At our center, ifosfamide-cisplatin combination chemotherapy is given for end-stage cervical cancer cases or recurrent cases that have been clinically established and supported by gyneco-oncologists. The regimen used is a combination of 2000 $\mathrm{mg} / \mathrm{m}^{2}$ ifosfamide and $30 \mathrm{mg} / \mathrm{m}^{2}$ cisplatin, given every three weeks. Chemotherapy is given inpatient, with the following conditions: (1) good performance score (Karnofsky score $>80$ ), (2) blood cell count: white blood cell count $\geq 3 \times 10^{3} / \mu \mathrm{L}$; hemoglobin $\geq 10 \mathrm{~g} / \mathrm{dL}$; platelets $\geq 100 \times 10^{3} / \mu \mathrm{L}$, (3) liver enzymes no more than twice the normal limit value (aspartate transferase $18.3 \mathrm{U} / \mathrm{L}$, alanine transferase $11.9 \mathrm{U} / \mathrm{L}$ ), (4) glomerular filtration rate $\geq 40 \mathrm{~mL} / \mathrm{min}$, (5) albumin serum $\geq 3 \mathrm{~g}$ / $\mathrm{dL},(6)$ other general parameters are good (normoglycemia, normokalemia, normonatremia).

During chemotherapy, premedication with dexamethason (10 mg intravenous in two doses), ondancentron ( $8 \mathrm{mg}$ intravenous single dose), hydration (2 liters of $0.9 \% \mathrm{NaCl}$ divided in half), and Mesna is given to overcome the toxicity of ifosfamide in vesica urinaria $(60 \%$ ifosfamide dose, in three divided doses, iv). Close monitoring is performed during ifosfamide administration, and if symptoms of a neurological disorder are found, consultation with a neurologist is carried out. Encephalopathy in patients is established by definition: fluctuations in the level of consciousness within hours to days, accompanied by changes in psychomotor activity (increasing or decreasing).

The degree of encephalopathy is graded using Meanwell criteria, which divides into 0 (conscious), 1 (transient lethargia), 2 (somnolens $<50 \%$ time and/or mild disorientation), 3 (somnolens $>50 \%$ time and/or severe disorientation, ecolalia, written perseveration, palilalia, logorea, hallucination or delusion), and 4 (comma). All patients agreed to written informed consent.

From 2015 to 2017, five patients received ifosfamide chemotherapy regimen at Sanglah Hospital Denpasar as a second line of chemotherapy, where the first line of chemotherapy was using paxus-carboplatin. Among the five patients, three were diagnosed with advanced stage and presented with kidney failure. One patient was diagnosed with inoperable stage IIB.

Before ifosfamide chemotherapy was given, these five patients had received 6 series of paxus-carboplatin chemotherapy, and some of them also had additional therapy in form of external radiation and brachytherapy. The chemotherapy regimen given for the second line was ifosfamide combined with cisplatin. The dose of ifosfamide giv- en had been adjusted based on the clinical and laboratory data of the patient, and the series given for each patient was varies, given at most five series, and at least as much as one series.

Table 1. Patient demographic data.

\begin{tabular}{cccccc}
\hline Patient & Age & $\begin{array}{c}\text { Marital } \\
\text { Age }\end{array}$ & $\begin{array}{c}\text { Ethnic } \\
\text { Group }\end{array}$ & $\begin{array}{c}\text { Number of } \\
\text { Parity }\end{array}$ & Preliminary Diagnosis \\
\hline 1 & 40 & 13 & Java & 3 & Ca cervix stage IIIB \\
\hline 2 & 47 & 18 & Bali & 2 & Ca Cervix stage IIB \\
\hline 3 & 59 & 18 & Bali & 3 & Ca Cervix stage IIB \\
\hline 4 & 42 & 18 & Bali & 2 & Ca cervix stage IIIB \\
\hline 5 & 41 & 15 & Java & 3 & Ca cervix stage IB2 progressive \\
\hline
\end{tabular}

Table 2. Patient medical history.

\begin{tabular}{cccc}
\hline Patient & $\begin{array}{c}\text { Preliminary } \\
\text { Diagnosis }\end{array}$ & Histopathology & Comorbidities \\
\hline 1 & Ca cervix stage IIIB & Non-Keratinizing & Acute Chronic Kidney \\
& Squamous Cell Carcinoma & Disease ec post renal \\
\hline 2 & Ca Cervix stage IIB & Non-Keratinizing & None \\
& & Squamous Cell Carcinoma & \\
\hline 3 & Ca Cervix stage IIB & Mucinous Adeno & Acute Chronic Kidney \\
& & Carcinoma & Disease ec suspek renal \\
\hline 4 & Ca cervix stage IIIB & Non-Keratinizing & Acute Chronic Kidney \\
& & Squamous Cell Carcinoma & Disease ec post renal \\
\hline 5 & Ca cervix stage IB2 & Non-Keratinizing & None \\
& progressive & Squamous Cell Carcinoma & \\
\hline
\end{tabular}

Monitoring was carried out every time a period / series of chemotherapy was completed given, and there was one patient who managed to "recover" with no side effects from ifosfamide and pap smear resulted in non-evidence of malignancy. Meanwhile, in the other four patients, side effects of ifosfamide such as transaminitis, kidney failure and encephalopathy appeared. The severity level of side effects appeared was different for each patient.

Patient who managed to recover was diagnosed with stage IIB without any comorbidities. This was different from other patients who came in advanced stages or even though they came in the early stage but the progression of the disease was rapid and was worsened by complications. Methylene blue as prophylaxis or encephalopathy therapy is not commonly given at Sanglah Hospital Denpasar, and for the establishment of encephalopathy diagnosis is based on clinical and laboratory symptoms only, in which EEG examination or CT scan and MRI are not routinely done. The treatment given for delirium was haloperidol. These side effects of encephalopathy occur usually within 6-24 hours after administration of an ifosfamide regimen.

Table 3. Treatment history and patient's outcome.

\begin{tabular}{cccccc}
\hline Patient & $\begin{array}{c}\text { First line } \\
\text { Chemotherapy } \\
\text { (Paxus } \\
\text { Carboplatin) }\end{array}$ & $\begin{array}{c}\text { Additional } \\
\text { Therapy }\end{array}$ & $\begin{array}{c}\text { Ifosfamide } \\
\text { Chemotherapy }\end{array}$ & Side Effects & Outcome \\
\hline 1 & 6 series & $\begin{array}{c}\text { External } \\
\text { Radiation 25x, } \\
\text { Brachyteraphy } \\
1 \mathrm{x}\end{array}$ & 1 series & $\begin{array}{c}\text { Encephalopathy, } \\
\text { transaminitis }\end{array}$ & Died \\
& & - & 1 series & None & "Recover' \\
\hline 2 & 6 series & $\begin{array}{c}\text { External } \\
\text { Radiation } 33 \mathrm{x}\end{array}$ & 5 series & $\begin{array}{c}\text { Transaminitis, } \\
\text { hypokalemia, } \\
\text { hipoalbumin }\end{array}$ & Died \\
\hline 3 & 6 series & - & 3 series & $\begin{array}{c}\text { Encephalopathy, } \\
\text { transaminitis }\end{array}$ & Died \\
\hline 4 & 6 series & - & 3 series & $\begin{array}{c}\text { Encephalopathy, } \\
\text { renal failure }\end{array}$ & Died \\
\hline 5 & 6 series & & & & \\
\hline
\end{tabular}




\section{Discussion}

It was estimated that about $35 \%$ of cervical cancer were recurrent or persistent, after receiving complete therapy. The recurrence rate was reported as $1.5 \%$ in stage $\mathrm{I}, 5 \%$ in stage $\mathrm{IIb}, 7.5 \%$ in stage IIIa, and $17 \%$ in stage IIIb. Most early-stage cancers were treated with surgery (with or without adjuvant), and advanced-stage cancer were treated with chemo radiation. Whereas, in recurrent or persistent cases, single dose chemotherapy was used in most centers. ${ }^{4}$ The chemotherapy regimen which commonly used in such cases were single cisplatin, which has been proven to show a response rate of $20-30 \%$. However, the duration of response to cisplatin and survival rates was still very low, especially in cases with platinum resistance. Thus, alternative chemotherapy agents were needed. Ifosfamide in a single dose gave a response rate of 33-60\%, while in combination TIP (paclitaxel-ifosfamid-cisplatin) gave a response of 52-75\% with a survival rate of more than 13 months in sensitive groups. ${ }^{2}$

Ifosfamide [3-(2-chloroethyl)-2-[(2-chloroethyl)-amino] tetrahydro-2H-1,3,2-oxazophosphorin-2-oxide] is an oxazophorine family and belongs to an alkylating agent. This agent is the result of chemical modification of cyclophosphamide, which is more soluble in water, and has better tumor activity and toxicity profiles. ${ }^{5}$ Ifosfamide is mostly dissolved in plasma fluid, and only a few is bound to carrier proteins. After a single intravenous injection, the elimination half-life of serum is around 4-8 hours, with wide interindividual variations. The drug is excreted in the urine around $14-53.1 \%$ at a rate of $0.66-$ $1.28 \mathrm{1} / \mathrm{hr}$, through feces less than $1 \%$, and the rest is metabolized. ${ }^{6}$

Ifosfamide and cyclophosphamide undergo bio activation by the cytochrome P450 system, and are metabolized through the N-dechloroethylation to chloroacetaldehyde (CAA). These metabolic products are estimated to have neurotoxic and nephrotoxic effects. However, ifosfamide is altered in greater amounts than cyclophosphamides (45\% vs $10 \%$ ), so it is more often related to neurotoxic effects. ${ }^{5}$ Side effects of ifosfamide are due to its metabolites in the body. Urological and renal tract toxicity are caused by acrolein metabolites while neurotoxicity is caused by CAA. Furthermore, ifosfamide is also easier to cross the blood-brain barrier than cyclophosphamide. ${ }^{7,8}$

Ifosfamide is known to cause cranial nerve paralysis, acute encephalopathy, and PRES syndrome. ${ }^{3}$ Both intravenous and oral administration provide similar outcomes, but oral routes are often related to neurotoxicity due to metabolic routes in the body. Encephalopathy was found in $10 \%$ of the intravenous route, and $50 \%$ of the oral route. ${ }^{8}$ Encephalopathy is generally the same as the term 'mental status change' by neurologists, and 'delirium' by psychiatrists. Encepalopathy is defined as general cortical dysfunction, which has an acute or sub-acute onset (hours to days), real fluctuation in level of consciousness, low attention, hallucinations and delusions that often arise, and changes in motoric activity (generally increasing, but at some time may decrease). Ifosfamide can cause encephalopathy-myoclonus syndrome. Sex (female), poor performance status, low albumin and hemoglobin levels, obesity, renal or hepatic dysfunction, and pleural or peritoneal effusion are risk factors for ifosfamide-induced encepalopathy. The dose of ifosfamide, the presence of tumor metastases to the brain, and age were not proven to be significant as a risk factor. ${ }^{9}$

Various pathways for the occurrence of neurotoxicity by ifosfamide had been studied, and most were considered to have links to ifosfamide metabolites, one of which is CCA. CCA can cross the blood-brain barrier and is thought to cause neurotoxicity through direct toxic effects on neuron cells, the decrease of glutathione in the central nervous system, or inhibition of mitochondrial oxidative phosphorylation, resulting in fatty acid metabolism disorder. Besides CCA, S-carboxymethylcysteine is also expected to cause neurotoxicity, through cell acidification and activation of specific receptors. ${ }^{10}$

Encephalopathy can occur during, immediately after, or until the fourth to sixth day after therapy begins. ${ }^{10}$ Acute symptoms were reported to occur within 2-146 hours after administration. Even so, 16 days of encephalopathy were reported after the combination of ifosfamide regimen given. The general symptoms were temporary and improving after 48-72 hours after discontinuation of therapy. Toxicity was reported to increase in short-term administration ( $<6$ hours). ${ }^{11}$

Altered consciousness is the most common symptom $(80 \%$ of cases) in the form of transient lethargy, drowsiness, to delirium. Other symptoms include hallucinations or psychosis $(30 \%)$, incontinence and muscle twitching ( $9 \%$ ), as well as other less common symptoms, such as extrapyramidal symptoms, dysarthria, cranial nerve disorders, seizures, mutism, and asterisks (5\%). Symptoms were generally mild and improve quickly, but the incidence of progressive encephalopathy that could not be treated and caused vegetative conditions and death had also been reported using Meanwell criteria, grade 2 encephalopathy was reported in $11 \%$ of cases, grade 3 in $77 \%$ of cases, and grade 4 in 11\% of cases. Lower degrees were usually not realized, and often considered to be a side effect of other drugs, such as antiemesis. ${ }^{10}$

The diagnosis of ifosfamide encephalopathy is clinical. Blood tests can show the presence of hypoalbuminemia, hypokalemia, hyponatremia, to kidney dysfunction. However, these results are not specific. Head imaging methods using magnetic resonance imaging (MRI) or computed tomography scan (CT-scan) do not show intracranial lesions and are usually done only if symptoms do not improve after the administration of ifosfamide is stopped. Electroencephalography (EEG) generally shows generalized irregular waves that are slowing down and interspersed with periodic triphasic waves when symptoms appear. At the third degree, it is usually found a dominant delta wave with or without the formation of sharp complex waves, while the fourth degree shows a continuous delta wave, and complex waves and triphasic waves. EEG abnormalities generally appear 12-24 hours before the onset of symptoms and improve 24-48 hours before clinical improvement. ${ }^{10}$

If the patient is suspected of having ifosfamid encephalopathy, then the drug administration must be stopped and a causal search 
must be carried out immediately. Hypoalbuminemia or hypokalemia should be corrected. Mild degree encepalopathy (1-2) generally does not require special therapy and will improve within 48-72 hours. Clinical monitoring should be carried out in this group, as well as adequate hydration to ensure renal function and metabolite excretion, temporary termination of ifosfamide, and supportive therapy. After stopped, ifosfamide can be given slowly through intravenous infusion for several days. Benzodiazepines for mild symptoms is not recommended because it can obscure important neurological changes. Therefore, haloperidol ( $1 \mathrm{mg}$ intravenously every 6 hours) can be given to treat symptoms of agitation or hallucination. ${ }^{12}$

If symptoms are severe (grade 3-4) or do not improve after stopping the drug, then methylene blue can be given. Methylene blue acts as an alternative electron acceptor and reverses the inhibition of NADH against gluconeogenesis, as well as inhibiting the transformation of chlorethylamine into CCA. Methylene blue also inhibits the activity of various amine oxidases, thus preventing the formation of CCA. ${ }^{13}$ Methylene blue $1 \%$ 50-60 mg solution can be given in the form of intravenous infusion for 5 minutes every four hours, until the patient's neurological status returns to normal. ${ }^{10,12}$ The administration of methylene blue as prophylaxis (50-100 mg intravenously or orally every 8 hours, the day before chemotherapy) had been reported, and had succeeded in preventing recurrent encepalopathy. ${ }^{12,14}$ However, this method has not been proven in larger studies.

It should be noted that the administration of methylene blue can cause side effects such as nausea, abdominal and precordial pain, dizziness, headache, excessive sweating, confusion, and methemoglobinemia. Thus, research to find safer alternatives is then undertaken. In some cases of ifosfamide encepalopathy, clinical symptoms and imaging results show disturbances in bilateral thalamus, similar to thiamine deficiency in Wernicke's encephalopathy. Research shows that administration of ifosfamide has not been proven to reduce levels of thiamine's active substance in the blood. However, ifosfamide and/or their metabolites are thought to compete with thiamine at their receptors. Thus, thiamine supplementation can interfere this balance in the direction of thiamine. Thiamine is given as much as $100 \mathrm{mg}$ through intravenous infusion every 4-6 hours, or orally 300 mg every 12 hours. Prophylactic protocols can also be considered in patients with risk factors, $100 \mathrm{mg}$ intravenously every 12 hours, begins 30 minutes before administration of ifosfamide, up to one day after therapy is completed. In cases that did not show a response to methylene blue, thiamine administration was reported to have successfully overcome symptoms on the second day of administration. Beside these therapies, there are several alternative therapies, namely albumin transfusion and oral Mesna, to increase CCA binding thereby preventing the entry of CCA into the central nerve. Hemodialysis can help the removal of metabolites from the body. When seizures occur, intravenous diazepam is reported to be effective in stopping episodes of seizures. ${ }^{10,15}$

\section{Conclusion}

Encepalopathy is a side effect that needs to be monitored in ifosfamide administration. Although most symptoms are mild and immediately improving after terminating ifosfamide, some cases can progressively worsen and become fatal. In mild degree encephalopathy, supportive monitoring and therapy is generally required. However, in severe cases, specific therapy is needed to improve the symptoms. Methylene blue and thiamine had been shown to be useful for treating encephalopathy in several studies although it has not yet been proven through larger studies. Both of these therapies are the first choice in cases of ifosfamide encephalopathy.

\section{References}

1. Nurcahyanti ADR. Cervical cancer: The case in Indonesia and natural product-based therapy. 2016:7.

2. Buda A, Dell'Anna T, Signorelli M, Mangioni C. Role of ifosfamide in cervical cancer: An overview. Oncology. 2003;65(2):63-6. DOI: $10.1159 / 000073362$

3. Livshits Z, Rao RB, Smith SW. An approach to chemotherapy-associated toxicity. Emerg Med Clin North Am. 2014;32(1):167-203. DOI 10.1016/j.emc.2013.09.002

4. Kumar L, Gupta S. Integrating chemotherapy in the management of cervical cancer: A critical appraisal. Oncology. 2016;91(1):8-17. DOI: $10.1159 / 000447576$

5. Tascilar M, Loos WJ, Seynaeve C, et al. The pharmacologic basis of ifosfamide use in adult patients with advanced soft tissue sarcomas. The Oncologist. 2007;12(11):1351-60. DOI: 10.1634/theoncologist.12-11-1351

6. Wagner T. Ifosfamide clinical pharmacokinetics. Clin Pharmacokinet. 1994;26(6):439-56. DOI: 10.2165/00003088-199426060-00003

7. Sleurs C, Deprez S, Emsell L, et al. Chemotherapy-induced neurotoxicity in pediatric solid non-CNS tumor patients: An update on current state of research and recommended future directions. Crit Rev Oncol Hematol. 2016;103:37-48. DOI: 10.1016/j.critrevonc.2016.05.001

8. Highley M, Momerency G, Sawyers D, et al. The neurotoxicity and pharmacokinetics of oral ifosfamide. J Anal Oncol. 2015;4(1):13-23. DOI: $10.6000 / 1927-7229.2015 .04 .01 .3$

9. Lo Y, Shen L-J, Chen W-H, et al. Risk factors of ifosfamide-related encephalopathy in adult patients with cancer: A retrospective analysis. J Formos Med Assoc. 2016;115(9):744-51. DOI: 10.1016/j. jfma.2015.07.016

10. Ajithkumar TV, Minimole AL, John MM, et al. Primary fallopian tube carcinoma. Obstet Gynecol Surv. 2005;60(4):247-52. DOI:10.1097/01. ogx.0000158506.23663.79

11. Alici-Evcimen Y, Breitbart WS. Ifosfamide neuropsychiatric toxicity in patients with cancer. Psychooncology. 2007;16(10):956-60. DOI 10.1002/pon.1161 
12. Patel PN. Methylene blue for management of ifosfamide-induced encephalopathy. Ann Pharmacother. 2006;40(2):299-303. DOI: 10.1345/ aph.1G114

13. Ginimuge PR, Jyothi SD. Methylene blue: revisited. J Anaesthesiol Clin Pharmacol. 2010;26(4):517-20.

14. Giovanis P, Garna A, Marcante M, et al. Ifosfamide encephalopathy and use of methylene blue. A case report of different sequential neurotoxicity. Tumori. 2009;95(4):545-46.

15. Lin JK, Chow DS, Sheu L, et al. Wernicke-like encephalopathy associated with ifosfamide. The Neurohospitalist. 2017;7(1):49-50. DOI: $10.1177 / 1941874416637407$ 